\title{
Kinetics and Mechanism of Ketone Enolization Mediated by Magnesium Bis(hexamethyldisilazide)
}

Xuyang He, J. Jacob Morris, Bruce C. Noll, Seth N. Brown ${ }^{*}$ and Kenneth W. Henderson* Department of Chemistry and Biochemistry, University of Notre Dame, Notre Dame, Indiana 46556, USA

\section{Supporting Information}

Table S1 Key bond lengths and angles for $(E)-\mathbf{1}$ and $(Z)-\mathbf{1}$.

Table S2 Rate constants of the enolizations in toluene

Table S3 Rate constants of the isotope effect in toluene

Table S4 Measured diffusion coefficients

Figure S1 Plot of absorbance versus time for the disappearance of intermediate 2

Figure S2 Eyring plot

Figure S3 $\quad{ }^{1} \mathrm{H}$ NMR spectra of free propiophenone and intermediate 2 in $\mathrm{CD}_{2} \mathrm{Cl}_{2}$

Tables of Cartesian coordinates and associated energies for the calculated geometries of: $\left\{\mathrm{Mg}(\mathrm{HMDS})_{2}\right\}_{2}(\mathbf{I}),(\mathrm{HMDS})_{2} \mathrm{MgO}=\mathrm{C}(\mathrm{Ph}) \mathrm{CH}_{2} \mathrm{CH}_{3}$ (II), $(\mathrm{HMDS})_{2} \mathrm{Mg}\left\{\mathrm{O}=\mathrm{C}(\mathrm{Ph}) \mathrm{CH}_{2} \mathrm{CH}_{3}\right\}_{2}$ (III), $\mathrm{O}=\mathrm{C}(\mathrm{Ph}) \mathrm{CH}_{2} \mathrm{CH}_{3}$ (IV) and $(\mathrm{HMDS})_{2} \mathrm{MgO}=\mathrm{C}\left({ }^{\mathrm{t}} \mathrm{Bu}\right) \mathrm{Ph}(\mathbf{V})$. 
Complete list of authors for reference 6c:

Pratt, L. M.; Newman, A.; St. Cyr, J.; Johnson, H.; Miles, B.; Lattier, A.; Austin, E.; Henderson, S.; Hershey, B.; Lin, M.; Balamraju, Y.; Sammonds, L.; Cheramie, J.; Karnes, J.; Hymel, E.; Woodford, B.; Carter, C. J. Org. Chem. 2003, 68, 6387.

Complete list of authors for reference 53:

M. J. Frisch, G. W. Trucks, H. B. Schlegel, G. E. Scuseria, M. A. Robb, J. R. Cheeseman, J. A. Montgomery, Jr., T. Vreven, K. N. Kudin, J. C. Burant, J. M. Millam, S. S. Iyengar, J. Tomasi, V. Barone, B. Mennucci, M. Cossi, G. Scalmani, N. Rega, G. A. Petersson, H. Nakatsuji, M. Hada, M. Ehara, K. Toyota, R. Fukuda, J. Hasegawa, M. Ishida, T.

Nakajima, Y. Honda, O. Kitao, H. Nakai, M. Klene, X. Li, J. E. Knox, H. P. Hratchian, J. B. Cross, C. Adamo, J. Jaramillo, R. Gomperts, R. E. Stratmann, O. Yazyev, A. J. Austin, R. Cammi, C. Pomelli, J. W. Ochterski, P. Y. Ayala, K. Morokuma, G. A. Voth, P. Salvador, J. J. Dannenberg, V. G. Zakrzewski, S. Dapprich, A. D. Daniels, M. C. Strain, O. Farkas, D. K. Malick, A. D. Rabuck, K. Raghavachari, J. B. Foresman, J. V. Ortiz, Q. Cui, A. G. Baboul, S. Clifford, J. Cioslowski, B. B. Stefanov, G. Liu, A. Liashenko, P. Piskorz, I. Komaromi, R. L. Martin, D. J. Fox, T. Keith, M. A. Al-Laham, C. Y. Peng, A. Nanayakkara, M. Challacombe, P. M. W. Gill, B. Johnson, W. Chen, M. W. Wong, C. Gonzalez, and J. A. Pople, Gaussian, Inc., Wallingford CT, 2004. 
Table S1. Key bond lengths $(\AA)$ and angles (deg) for $(E)-\mathbf{1}$ and $(Z)-\mathbf{1}$.

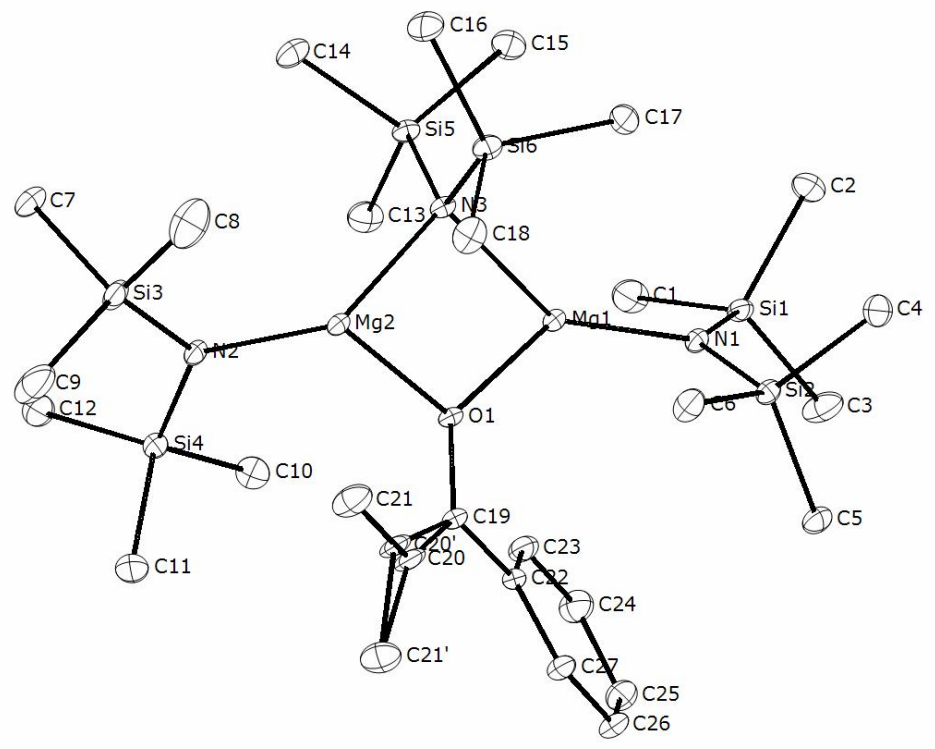

\begin{tabular}{|c|c|c|c|}
\hline $\mathrm{Mg}(1)-\mathrm{O}(1)$ & $1.9341(12)$ & $\operatorname{Mg}(1)-N(1)$ & $1.9452(14)$ \\
\hline $\operatorname{Mg}(1)-\mathrm{N}(3)$ & $2.1392(14)$ & $\operatorname{Mg}(1)-\operatorname{Mg}(2)$ & $2.9252(7)$ \\
\hline $\mathrm{Mg}(2)-\mathrm{O}(1)$ & $1.9426(12)$ & $\mathrm{Mg}(2)-\mathrm{N}(2)$ & $1.9463(15)$ \\
\hline $\operatorname{Mg}(2)-N(3)$ & $2.0997(14)$ & $\mathrm{N}(1)-\mathrm{Si}(1)$ & $1.7015(14)$ \\
\hline $\mathrm{N}(1)-\mathrm{Si}(2)$ & $1.7080(13)$ & $\mathrm{N}(2)-\mathrm{Si}(3)$ & $1.7086(16)$ \\
\hline $\mathrm{N}(2)-\mathrm{Si}(4)$ & $1.7165(17)$ & $\mathrm{N}(3)-\mathrm{Si}(5)$ & $1.7515(14)$ \\
\hline $\mathrm{N}(3)-\mathrm{Si}(6)$ & $1.7532(14)$ & $\mathrm{O}(1)-\mathrm{C}(19)$ & $1.3769(19)$ \\
\hline $\mathrm{Si}(1)-\mathrm{C}(3)$ & $1.8658(19)$ & $\mathrm{Si}(1)-\mathrm{C}(1)$ & $1.8681(19)$ \\
\hline $\mathrm{Si}(1)-\mathrm{C}(2)$ & $1.8696(19)$ & $\mathrm{Si}(2)-\mathrm{C}(5)$ & $1.8685(18)$ \\
\hline $\mathrm{Si}(2)-\mathrm{C}(4)$ & $1.8737(19)$ & $\mathrm{Si}(2)-\mathrm{C}(6)$ & $1.8781(18)$ \\
\hline $\operatorname{Si}(3)-C(7)$ & $1.8663(19)$ & $\mathrm{Si}(3)-\mathrm{C}(8)$ & $1.868(3)$ \\
\hline $\mathrm{Si}(3)-\mathrm{C}(9)$ & $1.869(2)$ & $\mathrm{Si}(4)-\mathrm{C}(10)$ & $1.869(2)$ \\
\hline $\mathrm{Si}(4)-\mathrm{C}(12)$ & $1.873(2)$ & $\mathrm{Si}(4)-\mathrm{C}(11)$ & $1.873(2)$ \\
\hline $\mathrm{Si}(5)-\mathrm{C}(14)$ & $1.8653(18)$ & $\mathrm{Si}(5)-\mathrm{C}(15)$ & $1.8676(19)$ \\
\hline $\mathrm{Si}(5)-\mathrm{C}(13)$ & $1.8708(19)$ & $\mathrm{Si}(6)-\mathrm{C}(16)$ & $1.8620(19)$ \\
\hline $\mathrm{Si}(6)-\mathrm{C}(17)$ & $1.8691(19)$ & $\mathrm{Si}(6)-\mathrm{C}(18)$ & $1.871(2)$ \\
\hline $\mathrm{O}(1)-\mathrm{Mg}(1)-\mathrm{N}(1)$ & $129.71(6)$ & $\mathrm{O}(1)-\mathrm{Mg}(1)-\mathrm{N}(3)$ & $86.57(5)$ \\
\hline $\mathrm{N}(1)-\mathrm{Mg}(1)-\mathrm{N}(3)$ & $143.30(6)$ & $\mathrm{O}(1)-\mathrm{Mg}(1)-\mathrm{Mg}(2)$ & $41.12(3)$ \\
\hline $\mathrm{N}(1)-\mathrm{Mg}(1)-\mathrm{Mg}(2)$ & $170.82(5)$ & $\mathrm{N}(3)-\mathrm{Mg}(1)-\mathrm{Mg}(2)$ & $45.80(4)$ \\
\hline $\mathrm{O}(1)-\mathrm{Mg}(2)-\mathrm{N}(2)$ & $128.59(6)$ & $\mathrm{O}(1)-\mathrm{Mg}(2)-\mathrm{N}(3)$ & $87.47(5)$ \\
\hline $\mathrm{N}(2)-\mathrm{Mg}(2)-\mathrm{N}(3)$ & $141.59(6)$ & $\mathrm{O}(1)-\mathrm{Mg}(2)-\mathrm{Mg}(1)$ & $40.90(4)$ \\
\hline $\mathrm{N}(2)-\mathrm{Mg}(2)-\mathrm{Mg}(1)$ & $161.52(6)$ & $\mathrm{N}(3)-\mathrm{Mg}(2)-\mathrm{Mg}(1)$ & $46.93(4)$ \\
\hline $\mathrm{Si}(1)-\mathrm{N}(1)-\mathrm{Si}(2)$ & $124.56(8)$ & $\mathrm{Si}(1)-\mathrm{N}(1)-\mathrm{Mg}(1)$ & $117.11(7)$ \\
\hline $\mathrm{Si}(2)-\mathrm{N}(1)-\mathrm{Mg}(1)$ & $118.28(7)$ & $\mathrm{Si}(3)-\mathrm{N}(2)-\mathrm{Si}(4)$ & $121.78(9)$ \\
\hline $\mathrm{Si}(3)-\mathrm{N}(2)-\mathrm{Mg}(2)$ & $117.96(9)$ & $\mathrm{Si}(4)-\mathrm{N}(2)-\mathrm{Mg}(2)$ & $119.70(8)$ \\
\hline $\mathrm{Si}(5)-\mathrm{N}(3)-\mathrm{Si}(6)$ & $117.85(8)$ & $\mathrm{Si}(5)-\mathrm{N}(3)-\mathrm{Mg}(2)$ & $108.45(7)$ \\
\hline $\operatorname{Si}(6)-N(3)-M g(2)$ & $118.69(7)$ & $\mathrm{Si}(5)-\mathrm{N}(3)-\mathrm{Mg}(1)$ & $118.49(7)$ \\
\hline $\operatorname{Si}(6)-N(3)-M g(1)$ & $102.49(6)$ & $\operatorname{Mg}(2)-N(3)-\operatorname{Mg}(1)$ & $87.27(5)$ \\
\hline $\mathrm{C}(19)-\mathrm{O}(1)-\mathrm{Mg}(1)$ & $131.96(10)$ & $\mathrm{C}(19)-\mathrm{O}(1)-\mathrm{Mg}(2)$ & $129.78(10)$ \\
\hline $\operatorname{Mg}(1)-\mathrm{O}(1)-\mathrm{Mg}(2)$ & $97.97(5)$ & $\mathrm{N}(1)-\mathrm{Si}(1)-\mathrm{C}(3)$ & $114.77(8)$ \\
\hline
\end{tabular}


Table S2. Rate constants for the enolization of propiophenone mediated by $\operatorname{Mg}(\mathrm{HMDS})_{2}$ in toluene at various temperatures and base concentrations. Pseudo-first-order conditions using a tenfold excess of base were applied. The rate constants quoted are the averages of values obtained from four measurements.

\begin{tabular}{|c|c|c|c|}
\hline Exp. No. & $\mathrm{T}\left({ }^{\circ} \mathrm{C}\right)$ & {$\left[\mathrm{Mg}(\mathrm{HMDS})_{2}\right](\mathrm{M})$} & $\mathrm{k}_{\mathrm{obs}}\left(\mathrm{s}^{-1}\right)$ \\
\hline 1 & 14.8 & $4.79 * 10^{-2}$ & $0.00234 \pm 0.00013$ \\
\hline 2 & 14.8 & $2.02 * 10^{-2}$ & $0.00197 \pm 0.00004$ \\
\hline 3 & 14.8 & $5.82 * 10^{-3}$ & $0.00432 \pm 0.00059$ \\
\hline 4 & 21.8 & $4.79 * 10^{-2}$ & $0.00499 \pm 0.00011$ \\
\hline 5 & 21.8 & $5.82 * 10^{-3}$ & $0.00514 \pm 0.00025$ \\
\hline 6 & 21.8 & $2.02 * 10^{-2}$ & $0.00499 \pm 0.00006$ \\
\hline 7 & 21.8 & $2.93 * 10^{-3}$ & $0.00457 \pm 0.00008$ \\
\hline 8 & 28.8 & $4.79 * 10^{-2}$ & $0.01171 \pm 0.00040$ \\
\hline 9 & 28.8 & $2.02 * 10^{-2}$ & $0.01082 \pm 0.00024$ \\
\hline 10 & 28.8 & $5.86 * 10^{-3}$ & $0.00953 \pm 0.00047$ \\
\hline 11 & 28.8 & $2.95 * 10^{-3}$ & $0.01126 \pm 0.0024$ \\
\hline 12 & 35.8 & $4.79 * 10^{-2}$ & $0.02042 \pm 0.00054$ \\
\hline 13 & 35.8 & $2.02 * 10^{-2}$ & $0.02033 \pm 0.00026$ \\
\hline 14 & 45.2 & $4.79 * 10^{-2}$ & $0.04279 \pm 0.00084$ \\
\hline 15 & 45.2 & $2.02 * 10^{-2}$ & $0.04069 \pm 0.00078$ \\
\hline
\end{tabular}

Table S3. Rate constants for the enolization of $\mathrm{O}=\mathrm{C}(\mathrm{Ph}) \mathrm{CD}_{2} \mathrm{CH}_{3}$ mediated by $\mathrm{Mg}(\mathrm{HMDS})_{2}$ in toluene at various temperatures. Pseudo-first-order conditions using a tenfold excess of base were applied. The rate constants quoted are the average values obtained from four measurements.

\begin{tabular}{|c|c|c|c|}
\hline Exp. No. & $\mathrm{T}\left({ }^{\circ} \mathrm{C}\right)$ & {$\left[\mathrm{Mg}(\mathrm{HMDS})_{2}\right](\mathrm{M})$} & $\mathrm{k}_{\mathrm{obs}}\left(\mathrm{s}^{-1}\right)$ \\
\hline 1 & 21.8 & $4.79 * 10^{-2}$ & $0.0002643 \pm 0.0000058$ \\
\hline 2 & 30.8 & $4.79 * 10^{-2}$ & $0.00101 \pm 0.00003$ \\
\hline 3 & 38.8 & $4.79 * 10^{-2}$ & $0.00249 \pm 0.00009$ \\
\hline 4 & 46.8 & $4.79 * 10^{-2}$ & $0.00559 \pm 0.00009$ \\
\hline 5 & 54.8 & $4.79 * 10^{-2}$ & $0.01106 \pm 0.00009$ \\
\hline 6 & 65.4 & $4.79 * 10^{-2}$ & $0.02249 \pm 0.00058$ \\
\hline
\end{tabular}


Table S4. Measured diffusion coefficients $(D)$ and the corresponding hydrodynamic radii $\left(r_{s}\right)$ for internal standard $\left[\mathrm{Mg}(\mathrm{HMDS})_{2}\right]_{2}$ and the pre-enolization complex 2.

\begin{tabular}{|c|c|c|c|c|}
\hline Sample & Molecule & $D\left(\mathrm{x} 10^{-11} \mathrm{~m}^{2} / \mathrm{s}\right)$ & $\mathrm{r}_{\mathrm{s}}(\AA)$ & $\mathrm{Vst}_{\mathrm{d}} / \mathrm{V}_{2}$ \\
\hline \multirow[t]{2}{*}{1 (run 1) } & {$\left[\mathrm{Mg}(\mathrm{HMDS})_{2}\right]_{2}$} & 9.362 & 1.66 & \multirow{2}{*}{1.33} \\
\hline & complex 2 & 10.30 & 1.51 & \\
\hline \multirow[t]{2}{*}{1 (run 2) } & {$\left[\mathrm{Mg}(\mathrm{HMDS})_{2}\right]_{2}$} & 8.306 & 1.87 & \multirow{2}{*}{1.26} \\
\hline & complex 2 & 8.993 & 1.73 & \\
\hline \multirow[t]{2}{*}{1 (run 3) } & {$\left[\mathrm{Mg}(\mathrm{HMDS})_{2}\right]_{2}$} & 7.617 & 2.04 & \multirow{2}{*}{1.24} \\
\hline & complex 2 & 8.199 & 1.90 & \\
\hline \multirow[t]{2}{*}{$2($ run 1$)$} & {$\left[\mathrm{Mg}(\mathrm{HMDS})_{2}\right]_{2}$} & 6.668 & 2.33 & \multirow{2}{*}{1.19} \\
\hline & complex $\mathbf{2}$ & 7.071 & 2.20 & \\
\hline \multirow[t]{2}{*}{2 (run 2) } & {$\left[\mathrm{Mg}(\mathrm{HMDS})_{2}\right]_{2}$} & 7.052 & 2.21 & \multirow{2}{*}{1.20} \\
\hline & complex $\mathbf{2}$ & 7.466 & 2.08 & \\
\hline \multirow[t]{2}{*}{2 (run 3$)$} & {$\left[\mathrm{Mg}(\mathrm{HMDS})_{2}\right]_{2}$} & 6.917 & 2.25 & \multirow{2}{*}{1.27} \\
\hline & complex 2 & 7.493 & 2.08 & \\
\hline
\end{tabular}


Figure S1. Plot of net absorbance at $330 \mathrm{~nm}$ versus time for the disappearance of intermediate 2 in toluene at $21.8^{\circ} \mathrm{C}$, with $\mathrm{Mg}(\operatorname{HMDS})_{2}(0.048 \mathrm{M})$ and propiophenone $(4.8$ $\left.\mathrm{x} 10^{-3} \mathrm{M}\right)$. The red line represents ideal first-order decay.

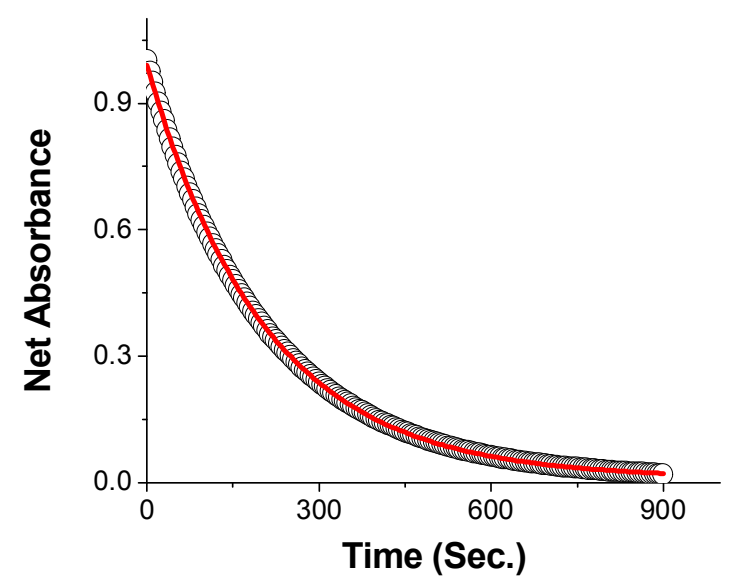


Figure S2. Eyring plot of the reaction of $\mathrm{Mg}(\mathrm{HMDS})_{2}(0.048 \mathrm{M})$ with $\mathrm{O}=\mathrm{C}(\mathrm{Ph}) \mathrm{CH}_{2} \mathrm{CH}_{3}$ $\left(4.8 \times 10^{-3} \mathrm{M}\right)$ in toluene.

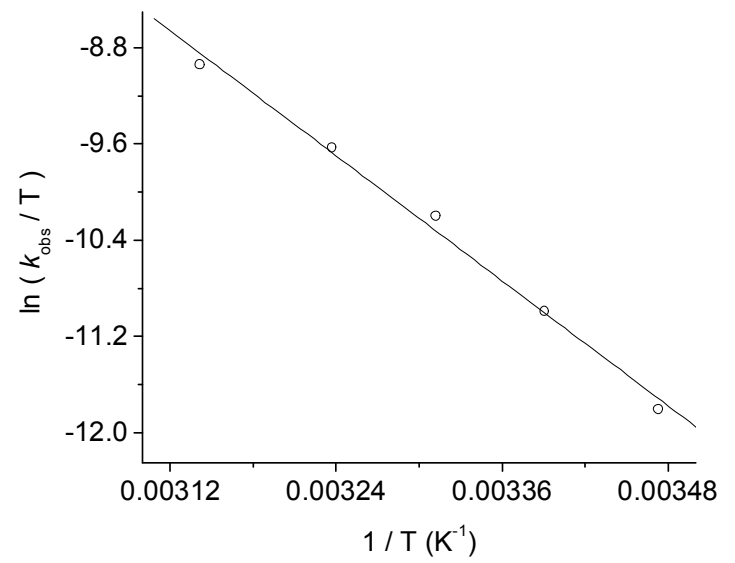


Figure S3. Comparison of ${ }^{1} \mathrm{H}$ NMR spectra of (a) free propiophenone and (b) the intermediate 2 at $-86^{\circ} \mathrm{C}$ in $\mathrm{CD}_{2} \mathrm{Cl}_{2}$.

(a)

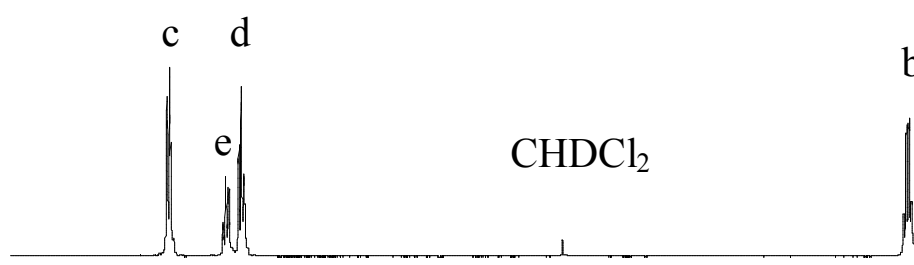

(b)

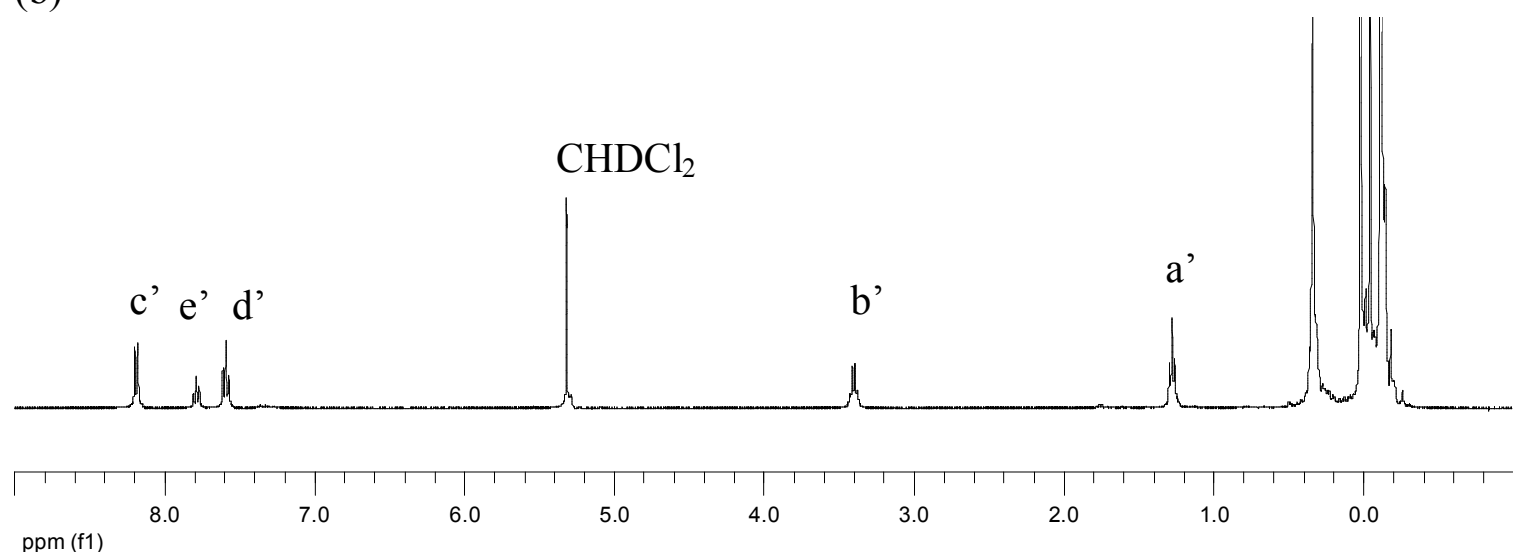

ppm (f1)<smiles>CCC(=O)c1ccccc1</smiles><smiles>CCC(=O)c1ccccc1</smiles> 


\section{$\left\{\operatorname{Mg}(\mathrm{HMDS})_{2}\right\}_{2}(\mathbf{I})$}

HF/6-31G* optimization: $-3879.67519074 \mathrm{~h}$ B3LYP/6-311++G ${ }^{* *}:-3894.29345262 \mathrm{~h}$

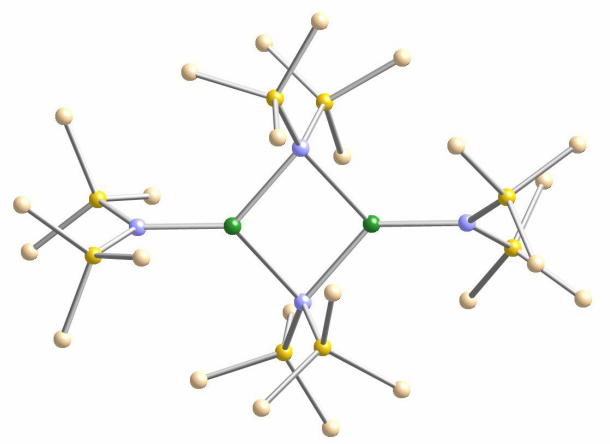

Cartesian coordinates for the $\mathrm{HF} / 6-31 \mathrm{G}^{*}$ minimum:

\begin{tabular}{|c|c|c|c|}
\hline $\mathrm{C}$ & -0.36835800 & -2.04823900 & -3.01087600 \\
\hline $\mathrm{C}$ & 0.49261700 & -4.39918300 & -1.36302300 \\
\hline $\mathrm{C}$ & 3.38610300 & -1.79489900 & -2.43750500 \\
\hline $\mathrm{C}$ & -2.28321800 & -3.33042800 & -1.09301700 \\
\hline $\mathrm{Si}$ & -0.48195900 & -2.77320600 & -1.2701050 \\
\hline $\mathrm{C}$ & -5.84715300 & -0.62229600 & -1.8263000 \\
\hline $\mathrm{C}$ & -3.29068400 & 0.45240400 & -2.9618540 \\
\hline $\mathrm{C}$ & 5.03303600 & 0.71231500 & -2.5271010 \\
\hline $\mathrm{C}$ & 5.89823900 & -1.68290600 & -0.8262980 \\
\hline $\mathrm{C}$ & 0.75448900 & 1.25148000 & -2.9177820 \\
\hline $\mathrm{Si}$ & 4.39978200 & -0.62709200 & -1.3364320 \\
\hline $\mathrm{Si}$ & -4.37234200 & 0.49959400 & -1.4005460 \\
\hline $\mathrm{N}$ & -0.00293500 & -1.62718700 & 0.0248060 \\
\hline $\mathrm{C}$ & 2.10950800 & -3.41335000 & 1.3861920 \\
\hline $\mathrm{C}$ & -0.82973500 & -3.71748500 & 2.1621830 \\
\hline $\mathrm{C}$ & -5.05679300 & -2.27014200 & 1.320667 \\
\hline $\mathrm{C}$ & -5.05685700 & 2.26992300 & -1.3205190 \\
\hline $\mathrm{Si}$ & 0.45877400 & -2.49105100 & 1.5155290 \\
\hline $\mathrm{N}$ & -3.48349900 & 0.00000000 & 0.0002700 \\
\hline $\mathrm{N}$ & 3.48661200 & 0.00000200 & 0.000173 \\
\hline $\mathrm{Mg}$ & -1.47131200 & -0.00010400 & -0.0000650 \\
\hline $\mathrm{Mg}$ & 1.47412400 & 0.00016700 & -0.000117 \\
\hline $\mathrm{C}$ & -0.82996400 & 3.71731800 & -2.1628350 \\
\hline $\mathrm{Si}$ & 0.45857700 & 2.49103300 & -1.515969 \\
\hline $\mathrm{C}$ & 2.10916500 & 3.41362500 & -1.3866210 \\
\hline $\mathrm{Si}$ & -4.37246400 & -0.49975000 & 1.400957 \\
\hline $\mathrm{N}$ & -0.00311000 & 1.62724700 & -0.0252110 \\
\hline $\mathrm{C}$ & 5.89751000 & 1.68368500 & 0.8271760 \\
\hline $\mathrm{Si}$ & 4.39942300 & 0.62718400 & 1.336991 \\
\hline $\mathrm{C}$ & 5.03322700 & -0.71209600 & 2.527505 \\
\hline $\mathrm{C}$ & 0.75427100 & -1.25160500 & 2.917541 \\
\hline $\mathrm{C}$ & -5.84742800 & 0.62202500 & 1.826497 \\
\hline $\mathrm{C}$ & -3.29114900 & -0.45265200 & 2.96249 \\
\hline $\mathrm{C}$ & -2.28334800 & 3.33083700 & 1.092355 \\
\hline $\mathrm{Si}$ & -0.48221600 & 2.77331100 & 1.2 \\
\hline $\mathrm{C}$ & 3.38506400 & 1.79428900 & 2.4 \\
\hline
\end{tabular}




\begin{tabular}{|c|c|c|c|}
\hline C & 0.49263900 & 4.39911800 & 1.36269300 \\
\hline C & -0.36901800 & 2.04808300 & 3.01032300 \\
\hline $\mathrm{H}$ & 4.07144700 & 2.37434000 & 3.05129600 \\
\hline $\mathrm{H}$ & 2.79239600 & 2.50456400 & 1.87295300 \\
\hline $\mathrm{H}$ & 2.72996400 & 1.27299600 & 3.12772300 \\
\hline $\mathrm{H}$ & 5.72801100 & 1.40475000 & -2.06387100 \\
\hline $\mathrm{H}$ & 4.21409600 & 1.30131200 & -2.93366600 \\
\hline $\mathrm{H}$ & 5.54633500 & 0.25209500 & -3.36973200 \\
\hline & 5.59009700 & 2.55592800 & 0.25483000 \\
\hline & 6.39664100 & 2.04714300 & 1.72343900 \\
\hline & 6.64341000 & 1.15701400 & 0.24153400 \\
\hline & 6.64406700 & -1.15573300 & -0.24101400 \\
\hline & 5.59114900 & -2.55495500 & -0.25348700 \\
\hline & 6.39735800 & -2.04665700 & -1.72244700 \\
\hline & 2.72907900 & -1.27421300 & -3.12562300 \\
\hline & 2.79554100 & -2.50683300 & -1.87209500 \\
\hline & 4.07277200 & -2.37303200 & -3.05207200 \\
\hline & 5.54527600 & -0.25162400 & 3.37076100 \\
\hline $\mathrm{H}$ & 5.72944200 & -1.40346600 & 2.06454300 \\
\hline $\mathrm{H}$ & 4.21466800 & -1.30226400 & 2.93312700 \\
\hline & 2.89906300 & -2.78737800 & 0.98299600 \\
\hline & 2.07077200 & -4.32002200 & 0.79750900 \\
\hline $\mathrm{H}$ & 2.41509800 & -3.70002200 & 2.39068700 \\
\hline & 1.39624300 & -0.41231700 & 2.66523800 \\
\hline $\mathrm{H}$ & -0.15373000 & -0.84727700 & 3.34379400 \\
\hline $\mathrm{H}$ & 1.26816000 & -1.77871500 & 3.71770800 \\
\hline $\mathrm{H}$ & 2.06995500 & 4.32085200 & -0.79881500 \\
\hline $\mathrm{H}$ & 2.41522500 & 3.69941000 & -2.39122400 \\
\hline $\mathrm{H}$ & 2.89864500 & 2.78818700 & -0.98244100 \\
\hline & -0.91444300 & 4.61142600 & -1.55446300 \\
\hline 11 & -1.82194700 & 3.28826000 & -2.25699600 \\
\hline $\mathrm{H}$ & -0.52251200 & 4.03943300 & -3.15580900 \\
\hline $\mathrm{H}$ & -2.45273200 & 3.92881200 & 0.20392600 \\
\hline 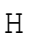 & -2.53126000 & 3.95682600 & 1.94779900 \\
\hline $\mathrm{H}$ & -2.99458500 & 2.51428200 & 1.07131400 \\
\hline $\mathrm{H}$ & 1.26703200 & 1.77903800 & -3.71852100 \\
\hline $\mathrm{H}$ & 1.39792200 & 0.41325300 & -2.66562500 \\
\hline $\mathrm{H}$ & -0.15323100 & 0.84569500 & -3.34322300 \\
\hline $\mathrm{H}$ & -2.33852700 & 0.96564300 & -2.85643000 \\
\hline 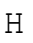 & -3.81277200 & 0.94804600 & -3.77726500 \\
\hline $\mathrm{H}$ & -3.09266200 & -0.56449600 & -3.28643000 \\
\hline $\mathrm{H}$ & -0.95868200 & -1.15590500 & -3.16518300 \\
\hline $\mathrm{H}$ & -0.77117600 & -2.80100900 & -3.68556500 \\
\hline $\mathrm{H}$ & 0.64229400 & -1.84303800 & -3.33595600 \\
\hline $\mathrm{H}$ & -6.63381100 & -0.61498000 & -1.07774300 \\
\hline $\mathrm{H}$ & -5.54090300 & -1.65560500 & -1.96864400 \\
\hline $\mathrm{H}$ & -6.29559200 & -0.28685400 & -2.75966400 \\
\hline $\mathrm{H}$ & -2.99433800 & -2.51378300 & -1.07156300 \\
\hline $\mathrm{H}$ & -2.45263700 & -3.92875400 & -0.20482500 \\
\hline $\mathrm{H}$ & -2.53130000 & -3.95600900 & -1.94870800 \\
\hline $\mathrm{H}$ & 1.56152000 & -4.28417600 & -1.49185300 \\
\hline $\mathrm{H}$ & 0.12024800 & -4.92009800 & -2.24319800 \\
\hline $\mathrm{H}$ & 0.32524000 & -5.05960100 & -0.51937100 \\
\hline $\mathrm{H}$ & -5.70969200 & -2.43275900 & 0.46927700 \\
\hline $\mathrm{H}$ & -4.26710900 & -3.01361700 & 1.26591500 \\
\hline $\mathrm{H}$ & -5.63642900 & -2.48152300 & 2.21755300 \\
\hline & -1.82171800 & -3.28841500 & 2.25632700 \\
\hline
\end{tabular}




$\begin{array}{rrrr}\mathrm{H} & -0.52233900 & -4.03965200 & 3.15515900 \\ \mathrm{H} & -0.91421100 & -4.61155400 & 1.55375900 \\ \mathrm{H} & -3.09282600 & 0.56421700 & 3.28697700 \\ \mathrm{H} & -2.33916200 & -0.96626100 & 2.85736700 \\ \mathrm{H} & -3.81364800 & -0.94798200 & 3.77782900 \\ \mathrm{H} & -5.54120300 & 1.65530400 & 1.96913200 \\ \mathrm{H} & -6.63386100 & 0.61487000 & 1.07770300 \\ \mathrm{H} & -6.29615300 & 0.28641400 & 2.75966200 \\ \mathrm{H} & -4.26726200 & 3.01352100 & -1.26616500 \\ \mathrm{H} & -5.70954200 & 2.43265800 & -0.46898700 \\ \mathrm{H} & -5.63676800 & 2.48100500 & -2.21729700 \\ \mathrm{H} & -0.77290800 & 2.80030200 & 3.68498600 \\ \mathrm{H} & -0.95858000 & 1.15515600 & 3.16407100 \\ \mathrm{H} & 0.64164700 & 1.84372400 & 3.33589300 \\ \mathrm{H} & 0.12104000 & 4.91965700 & 2.24341700 \\ \mathrm{H} & 0.32451800 & 5.05990100 & 0.51946000 \\ \mathrm{H} & 1.56164800 & 4.28407300 & 1.49052400\end{array}$

\section{Cartesian coordinates for the B3LYP/6-311+ $+\mathrm{G}^{* *}$ minimum:}

$\begin{array}{lrrr}\text { C } & 0.43330500 & 1.95172300 & -3.03506900 \\ \text { C } & -0.47443500 & 4.34969700 & -1.47913600 \\ \text { C } & -3.34900300 & 1.81352800 & -2.39336500 \\ \text { C } & 2.30769500 & 3.27799300 & -1.10752900 \\ \text { Si } & 0.50880600 & 2.73831400 & -1.32155900 \\ \text { C } & 5.84490600 & 0.56156100 & -1.83384900 \\ \text { C } & 3.28607900 & -0.56110600 & -2.94720500 \\ \text { C } & -4.94587700 & -0.75081000 & -2.55709800 \\ \text { C } & -5.93116500 & 1.61534600 & -0.89810900 \\ \text { C } & -0.72046000 & -1.32392900 & -2.94845300 \\ \text { Si } & -4.38538000 & 0.61209500 & -1.36101000 \\ \text { Si } & 4.37234000 & -0.55306500 & -1.39075400 \\ \text { N } & 0.00172300 & 1.61779300 & 0.00434400 \\ \text { C } & -2.13281800 & 3.41491800 & 1.30596600 \\ \text { C } & 0.80697200 & 3.80136500 & 2.06600300 \\ \text { C } & 5.07589400 & 2.27686400 & 1.27390000 \\ \text { C } & 5.06156200 & -2.31451500 & -1.24234100 \\ \text { Si } & -0.46868600 & 2.53282400 & 1.48092200 \\ \text { N } & 3.47293600 & -0.00883000 & 0.00531800 \\ \text { N } & -3.48456100 & 0.00197000 & 0.00890800 \\ \text { Mg } & 1.46315200 & 0.00065600 & 0.00029100 \\ \text { Mg } & -1.47478100 & 0.00793600 & 0.00245800 \\ \text { C } & 0.81509600 & -3.77581500 & -2.07580500 \\ \text { Si } & -0.46792800 & -2.51142400 & -1.49802500 \\ \text { C } & -2.13064000 & -3.39964900 & -1.34038200 \\ \text { Si } & 4.36112800 & 0.52481300 & 1.41262000 \\ \text { N } & -0.00789100 & -1.60509300 & -0.01288000 \\ \text { C } & -5.86225200 & -1.72809100 & 0.83961800 \\ \text { Si } & -4.41050200 & -0.61687600 & 1.35750900 \\ \text { C } & -5.10888700 & 0.74687100 & 2.47838700 \\ \text { C } & -0.73212200 & 1.35459700 & 2.93659900 \\ \text { C } & 5.81178000 & -0.60918400 & 1.87678900 \\ \text { C } & 3.25093600 & 0.54917600 & 2.95202600 \\ \text { C } & 2.27060500 & -3.28081800 & 1.13676300 \\ \text { Si } & 0.47183200 & -2.72991200 & 1.31873200\end{array}$




\begin{tabular}{|c|c|c|c|}
\hline C & -3.36735500 & -1.70422900 & 2.50651000 \\
\hline C & -0.52444500 & -4.33605600 & 1.45163100 \\
\hline C & 0.35837000 & -1.94034500 & 3.02766700 \\
\hline $\mathrm{H}$ & -4.03916000 & -2.22974500 & 3.19342400 \\
\hline $\mathrm{H}$ & -2.79953200 & -2.46469100 & 1.96764800 \\
\hline $\mathrm{H}$ & -2.67789700 & -1.12991600 & 3.12936900 \\
\hline & -5.63054500 & -1.45901500 & -2.08385400 \\
\hline & -4.09669700 & -1.32313400 & -2.94237400 \\
\hline & -5.46480400 & -0.31335300 & -3.41729300 \\
\hline & -5.51382400 & -2.60692500 & 0.28828000 \\
\hline & -6.38273400 & -2.08559000 & 1.73489300 \\
\hline & -6.60233500 & -1.21887200 & 0.21805300 \\
\hline & -6.68032200 & 1.04303500 & -0.34627600 \\
\hline & -5.67336000 & 2.49286500 & -0.29766400 \\
\hline & -6.41040700 & 1.97339900 & -1.81608000 \\
\hline $\mathrm{H}$ & -2.50021800 & 1.34172500 & -2.89245100 \\
\hline $\mathrm{H}$ & -2.97463200 & 2.64280900 & -1.78992200 \\
\hline $\mathrm{H}$ & -3.97614100 & 2.23958000 & -3.18347600 \\
\hline $\mathrm{H}$ & -5.61162600 & 0.30448600 & 3.34575900 \\
\hline $\mathrm{H}$ & -5.83325700 & 1.38519100 & 1.96732200 \\
\hline $\mathrm{H}$ & -4.31088900 & 1.39367000 & 2.85622900 \\
\hline $\mathrm{H}$ & -2.92606900 & 2.71785900 & 1.02299500 \\
\hline $\mathrm{H}$ & -2.12901400 & 4.23495500 & 0.58856100 \\
\hline 11 & -2.40183800 & 3.83507700 & 2.28163600 \\
\hline $\mathrm{H}$ & -1.43258200 & 0.53632400 & 2.74842300 \\
\hline $\mathrm{H}$ & 0.18806800 & 0.92351000 & 3.32936900 \\
\hline $\mathrm{H}$ & -1.17910000 & 1.94419400 & 3.74408600 \\
\hline $\mathrm{H}$ & -2.11591200 & -4.24858700 & -0.65768100 \\
\hline $\mathrm{H}$ & -2.41170400 & -3.77987300 & -2.32885400 \\
\hline $\mathrm{H}$ & -2.92139400 & -2.71696900 & -1.01882700 \\
\hline $\mathrm{H}$ & 0.90968800 & -4.62949300 & -1.40116300 \\
\hline $\mathrm{H}$ & 1.80954500 & -3.34383400 & -2.21019100 \\
\hline $\mathrm{H}$ & 0.49369000 & -4.16766200 & -3.04709100 \\
\hline $\mathrm{H}$ & 2.42806600 & -3.90934200 & 0.25826300 \\
\hline $\mathrm{H}$ & 2.54437400 & -3.87433100 & 2.01604700 \\
\hline $\mathrm{H}$ & 2.96714600 & -2.44276700 & 1.06820500 \\
\hline $\mathrm{H}$ & -1.13866000 & -1.91356300 & -3.77114700 \\
\hline $\mathrm{H}$ & -1.44069800 & -0.52090200 & -2.76756700 \\
\hline $\mathrm{H}$ & 0.19958400 & -0.87274500 & -3.31777100 \\
\hline $\mathrm{H}$ & 2.34696200 & -1.10914500 & -2.82674700 \\
\hline $\mathrm{H}$ & 3.82888100 & -1.05819300 & -3.75817700 \\
\hline $\mathrm{H}$ & 3.05694600 & 0.45105200 & -3.28919200 \\
\hline $\mathrm{H}$ & 1.02721100 & 1.04479200 & -3.13780600 \\
\hline $\mathrm{H}$ & 0.86094000 & 2.68315900 & -3.72972000 \\
\hline $\mathrm{H}$ & -0.57858500 & 1.73829400 & -3.37764300 \\
\hline $\mathrm{H}$ & 6.62667700 & 0.56315700 & -1.06931900 \\
\hline $\mathrm{H}$ & 5.53606600 & 1.59848400 & -1.99568300 \\
\hline $\mathrm{H}$ & 6.30428400 & 0.20452400 & -2.76228400 \\
\hline $\mathrm{H}$ & 2.99777200 & 2.43482500 & -1.03646200 \\
\hline $\mathrm{H}$ & 2.45642100 & 3.89829400 & -0.22187800 \\
\hline $\mathrm{H}$ & 2.59786600 & 3.87689300 & -1.97785300 \\
\hline $\mathrm{H}$ & -1.53722200 & 4.20233200 & -1.67429700 \\
\hline $\mathrm{H}$ & -0.05561600 & 4.87899000 & -2.34237200 \\
\hline $\mathrm{H}$ & -0.37038600 & 5.01225400 & -0.61816600 \\
\hline $\mathrm{H}$ & 5.75834100 & 2.37974600 & 0.42707800 \\
\hline $\mathrm{H}$ & 4.29334500 & 3.03092400 & 1.15670600 \\
\hline & 5.63794100 & 2.52132300 & 2.1822140 \\
\hline
\end{tabular}




$\begin{array}{rrrr}\mathrm{H} & 1.79955100 & 3.36952300 & 2.21442700 \\ \mathrm{H} & 0.47362500 & 4.19608200 & 3.03212500 \\ \mathrm{H} & 0.91008500 & 4.65285500 & 1.39001300 \\ \mathrm{H} & 2.99618200 & -0.45889000 & 3.28803100 \\ \mathrm{H} & 2.32487900 & 1.11602600 & 2.81784800 \\ \mathrm{H} & 3.78994600 & 1.03390600 & 3.77295800 \\ \mathrm{H} & 5.48648300 & -1.64116400 & 2.03768800 \\ \mathrm{H} & 6.60125500 & -0.62360000 & 1.12050400 \\ \mathrm{H} & 6.26601800 & -0.25598100 & 2.80916700 \\ \mathrm{H} & 4.26747700 & -3.05787300 & -1.13431100 \\ \mathrm{H} & 5.73207500 & -2.42587400 & -0.38706700 \\ \mathrm{H} & 5.63131800 & -2.56738700 & -2.14352800 \\ \mathrm{H} & 0.76402900 & -2.67027800 & 3.73687700 \\ \mathrm{H} & 0.95128000 & -1.03419400 & 3.14311500 \\ \mathrm{H} & -0.66321300 & -1.72351200 & 3.33747600 \\ \mathrm{H} & -0.14435000 & -4.85933100 & 2.33614000 \\ \mathrm{H} & -0.38521600 & -5.00560600 & 0.60090500 \\ \mathrm{H} & -1.59495500 & -4.18707100 & 1.59833400\end{array}$

\section{$(\mathrm{HMDS}){ }_{2} \mathrm{Mg}\left\{\mathrm{O}=\mathrm{C}(\mathrm{Ph}) \mathrm{CH}_{2} \mathrm{CH}_{3}\right\}$ (II)}

\section{$\mathrm{HF} / 6-31 \mathrm{G}^{*}$ optimization: $-2361.38355216 \mathrm{~h}$ B3LYP/6-311++G** : $-2371.49587071 \mathrm{~h}$}

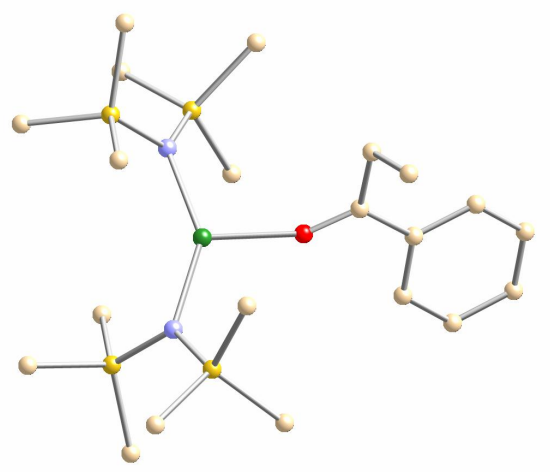

\section{Cartesian coordinates for the $\mathrm{HF} / 6-31 \mathrm{G}^{*}$ minimum:}

$\begin{array}{lrrr}\mathrm{Mg} & 0.58232000 & 0.02727200 & 0.07111400 \\ \mathrm{Si} & 0.50219800 & 3.02692800 & 1.12467300 \\ \mathrm{Si} & 1.95400100 & 2.43452600 & -1.47711600 \\ \mathrm{Si} & 0.63581300 & -2.97317700 & -1.02790400 \\ \mathrm{Si} & 2.78088500 & -2.07845300 & 0.92703700 \\ \mathrm{~N} & 1.05253100 & 1.95312500 & -0.09477200 \\ \mathrm{~N} & 1.37235200 & -1.79731900 & -0.01852300 \\ \mathrm{C} & 0.25583500 & 2.10014400 & 2.77276400 \\ \mathrm{C} & -1.17439900 & 3.82413600 & 0.70639400 \\ \mathrm{C} & 1.69855500 & 4.44476500 & 1.53368700 \\ \mathrm{C} & 1.24964800 & 3.97210900 & -2.34806900 \\ \mathrm{C} & 3.78393700 & 2.78132000 & -1.10808900 \\ \mathrm{C} & 1.92398300 & 1.05252700 & -2.78350200 \\ \mathrm{C} & 1.86116400 & -4.07178400 & -1.97657500 \\ \mathrm{C} & -0.43858800 & -2.14731100 & -2.36704700\end{array}$




\begin{tabular}{|c|c|c|c|}
\hline C & -0.52567600 & -4.14710900 & -0.07875000 \\
\hline $\mathrm{C}$ & 2.74532200 & -3.72319300 & 1.88127000 \\
\hline C & 2.94944600 & -0.71934500 & 2.24730800 \\
\hline 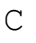 & 4.39168800 & -2.04514100 & -0.07762700 \\
\hline 0 & -1.41801200 & -0.12211900 & 0.43678300 \\
\hline $\mathrm{H}$ & -0.35156600 & 1.20170100 & 2.67699500 \\
\hline $\mathrm{H}$ & -0.24418600 & 2.73952400 & 3.49778900 \\
\hline $\mathrm{H}$ & 1.20662600 & 1.80380400 & 3.20704200 \\
\hline $\mathrm{H}$ & -1.50609800 & 4.49693100 & 1.49516100 \\
\hline $\mathrm{H}$ & -1.95084600 & 3.07313000 & 0.57281500 \\
\hline $\mathrm{H}$ & -1.12441000 & 4.40147000 & -0.21344300 \\
\hline $\mathrm{H}$ & 1.29674400 & 5.05141400 & 2.34325100 \\
\hline $\mathrm{H}$ & 1.87620700 & 5.11124800 & 0.69410900 \\
\hline $\mathrm{H}$ & 2.66411300 & 4.06664100 & 1.86021400 \\
\hline $\mathrm{H}$ & 0.22088100 & 3.80955400 & -2.66280500 \\
\hline $\mathrm{H}$ & 1.82887500 & 4.20486600 & -3.23961800 \\
\hline $\mathrm{H}$ & 1.25996500 & 4.85943400 & -1.72046400 \\
\hline $\mathrm{H}$ & 4.33261300 & 3.00826900 & -2.02034900 \\
\hline $\mathrm{H}$ & 4.26235500 & 1.91987100 & -0.64687900 \\
\hline $\mathrm{H}$ & 3.91517300 & 3.62256000 & -0.43312500 \\
\hline $\mathrm{H}$ & 2.52002700 & 1.33998000 & -3.64714500 \\
\hline $\mathrm{H}$ & 0.91574300 & 0.86090700 & -3.14755100 \\
\hline $\mathrm{H}$ & 2.33137200 & 0.11108800 & -2.42086500 \\
\hline $\mathrm{H}$ & 1.32535700 & -4.76186800 & -2.62569700 \\
\hline $\mathrm{H}$ & 2.48964200 & -4.67124700 & -1.32326000 \\
\hline $\mathrm{H}$ & 2.51915800 & -3.47786000 & -2.60616200 \\
\hline $\mathrm{H}$ & -0.96151100 & -2.90665900 & -2.94510200 \\
\hline $\mathrm{H}$ & 0.16484800 & -1.57495500 & -3.06651900 \\
\hline $\mathrm{H}$ & -1.20259100 & -1.48235300 & -1.96848100 \\
\hline $\mathrm{H}$ & 0.00148400 & -4.70205900 & 0.69264100 \\
\hline $\mathrm{H}$ & -0.98727100 & -4.87304300 & -0.74549800 \\
\hline $\mathrm{H}$ & -1.33085300 & -3.60318500 & 0.41227400 \\
\hline $\mathrm{H}$ & 1.88409600 & -3.77834900 & 2.54371600 \\
\hline $\mathrm{H}$ & 3.63643200 & -3.82486500 & 2.49772800 \\
\hline $\mathrm{H}$ & 2.70871600 & -4.59161700 & 1.22848600 \\
\hline $\mathrm{H}$ & 3.84469400 & -0.88351200 & 2.84339300 \\
\hline $\mathrm{H}$ & 2.10794900 & -0.71461300 & 2.93863800 \\
\hline $\mathrm{H}$ & 3.03622700 & 0.27865100 & 1.82227900 \\
\hline $\mathrm{H}$ & 5.26020600 & -2.15590500 & 0.56907800 \\
\hline $\mathrm{H}$ & 4.50317000 & -1.10432700 & -0.61247600 \\
\hline $\mathrm{H}$ & 4.43293900 & -2.84174300 & -0.81532600 \\
\hline $\mathrm{C}$ & -2.49783400 & -0.52751800 & 0.82214600 \\
\hline $\mathrm{C}$ & -3.70242500 & -0.28789800 & -0.00809900 \\
\hline C & -3.60825900 & 0.59585700 & -1.08435100 \\
\hline $\mathrm{C}$ & -4.70751700 & 0.84114500 & -1.88337700 \\
\hline $\mathrm{C}$ & -5.90877500 & 0.19860500 & -1.62159900 \\
\hline $\mathrm{C}$ & -6.01344600 & -0.68458200 & -0.55853400 \\
\hline C & -4.91659900 & -0.92335100 & 0.24870000 \\
\hline $\mathrm{H}$ & -2.67740600 & 1.09129200 & -1.28011800 \\
\hline $\mathrm{H}$ & -4.62961300 & 1.52782700 & -2.70578200 \\
\hline $\mathrm{H}$ & -6.76332900 & 0.38644600 & -2.24622100 \\
\hline $\mathrm{H}$ & -6.94350100 & -1.18433200 & -0.35992700 \\
\hline $\mathrm{H}$ & -5.01459600 & -1.61200300 & 1.06569400 \\
\hline $\mathrm{C}$ & -2.59147500 & -1.23420000 & 2.15453900 \\
\hline $\mathrm{H}$ & -3.15107300 & -2.15497600 & 2.04059900 \\
\hline $\mathrm{H}$ & -1.58375800 & -1.50147600 & 2.44220400 \\
\hline C & -3.23774600 & -0.35346900 & 3.2372690 \\
\hline
\end{tabular}




$\begin{array}{lrrr}\mathrm{H} & -3.23557200 & -0.88639900 & 4.18059600 \\ \mathrm{H} & -2.6844400 & 0.56780300 & 3.37384200 \\ \mathrm{H} & -4.26249500 & -0.09981000 & 2.99530700\end{array}$

\section{Cartesian coordinates for the B3LYP/6-311++G ${ }^{* *}$ minimum:}

\begin{tabular}{|c|c|c|c|}
\hline $\mathrm{Mg}$ & 0.58196200 & 0.02320200 & 0.05124400 \\
\hline $\mathrm{Si}$ & 0.65356000 & 3.04209700 & 1.10533600 \\
\hline $\mathrm{Si}$ & 2.26212400 & 2.30018300 & -1.39375600 \\
\hline $\mathrm{Si}$ & 0.49292000 & -2.95025700 & -1.16143800 \\
\hline $\mathrm{Si}$ & 2.53689300 & -2.26240000 & 1.02098800 \\
\hline $\mathrm{N}$ & 1.20769300 & 1.90459500 & -0.07441600 \\
\hline $\mathrm{N}$ & 1.21956600 & -1.85931200 & -0.03202200 \\
\hline C & 0.17921500 & 2.14182100 & 2.71367000 \\
\hline $\mathrm{C}$ & -0.89587200 & 3.98574000 & 0.53944800 \\
\hline C & 1.94494400 & 4.33955000 & 1.60608500 \\
\hline C & 1.77516600 & 3.89787600 & -2.29994000 \\
\hline C & 4.07702200 & 2.47832200 & -0.87143300 \\
\hline C & 2.19808400 & 0.91579000 & -2.69259600 \\
\hline C & 1.74428700 & -3.99987300 & -2.12625900 \\
\hline C & -0.51088600 & -1.98594000 & -2.45879200 \\
\hline $\mathrm{C}$ & -0.72465100 & -4.16215900 & -0.34352500 \\
\hline C & 2.33622300 & -3.94412200 & 1.88109700 \\
\hline C & 2.64509800 & -0.95756900 & 2.39851400 \\
\hline C & 4.22051600 & -2.27737300 & 0.14829100 \\
\hline O & -1.44948600 & -0.00216500 & 0.35291300 \\
\hline $\mathrm{H}$ & -0.58103200 & 1.37025500 & 2.55409500 \\
\hline $\mathrm{H}$ & -0.23604800 & 2.85140400 & 3.43736800 \\
\hline $\mathrm{H}$ & 1.04732800 & 1.66705100 & 3.18013800 \\
\hline $\mathrm{H}$ & -1.24211000 & 4.68988500 & 1.30412800 \\
\hline $\mathrm{H}$ & -1.71893900 & 3.29571600 & 0.32570200 \\
\hline $\mathrm{H}$ & -0.70041800 & 4.55615100 & -0.37351200 \\
\hline $\mathrm{H}$ & 1.53582800 & 4.99895600 & 2.37935200 \\
\hline $\mathrm{H}$ & 2.25211500 & 4.97291600 & 0.76885600 \\
\hline $\mathrm{H}$ & 2.84481400 & 3.86892400 & 2.01354400 \\
\hline $\mathrm{H}$ & 0.74928300 & 3.84282500 & -2.67800500 \\
\hline $\mathrm{H}$ & 2.43578000 & 4.06567600 & -3.15771800 \\
\hline $\mathrm{H}$ & 1.84530200 & 4.78261400 & -1.65992400 \\
\hline $\mathrm{H}$ & 4.71899900 & 2.68655900 & -1.73455900 \\
\hline $\mathrm{H}$ & 4.44240200 & 1.56021800 & -0.40021000 \\
\hline $\mathrm{H}$ & 4.21343600 & 3.29230700 & -0.15373500 \\
\hline $\mathrm{H}$ & 2.91545100 & 1.11954700 & -3.49465000 \\
\hline $\mathrm{H}$ & 1.20896400 & 0.84355900 & -3.15789900 \\
\hline $\mathrm{H}$ & 2.45224700 & -0.06608800 & -2.27911300 \\
\hline $\mathrm{H}$ & 1.23281400 & -4.64221300 & -2.85140100 \\
\hline $\mathrm{H}$ & 2.32968900 & -4.65225900 & -1.47121500 \\
\hline $\mathrm{H}$ & 2.44733800 & -3.36894500 & -2.67834300 \\
\hline $\mathrm{H}$ & -1.03293400 & -2.68464200 & -3.12139700 \\
\hline $\mathrm{H}$ & 0.13193800 & -1.36312700 & -3.08793300 \\
\hline $\mathrm{H}$ & -1.27840700 & -1.34087300 & -2.01722200 \\
\hline $\mathrm{H}$ & -0.22902600 & -4.78014100 & 0.41072200 \\
\hline $\mathrm{H}$ & -1.17212300 & -4.83553100 & -1.08292700 \\
\hline $\mathrm{H}$ & -1.54160900 & -3.63035600 & 0.15573000 \\
\hline $\mathrm{H}$ & 1.40615100 & -3.99116900 & 2.45635000 \\
\hline $\mathrm{H}$ & 3.16561900 & -4.11855100 & 2.57522400 \\
\hline
\end{tabular}




$\begin{array}{lrrr}\mathrm{H} & 2.32868800 & -4.77687000 & 1.17096600 \\ \mathrm{H} & 3.50015600 & -1.16349600 & 3.05108100 \\ \mathrm{H} & 1.75127700 & -0.95524700 & 3.03232300 \\ \mathrm{H} & 2.78628900 & 0.05556700 & 2.00551800 \\ \mathrm{H} & 5.03000900 & -2.50246000 & 0.85145300 \\ \mathrm{H} & 4.43743300 & -1.30586800 & -0.30697600 \\ \mathrm{H} & 4.25514600 & -3.02697400 & -0.64751200 \\ \mathrm{C} & -2.54313500 & -0.43946800 & 0.73030000 \\ \mathrm{C} & -3.76455800 & -0.08111400 & -0.02480000 \\ \mathrm{C} & -3.67334800 & 0.88924100 & -1.03808000 \\ \mathrm{C} & -4.79627100 & 1.24623700 & -1.77111000 \\ \mathrm{C} & -6.02417200 & 0.63604800 & -1.50815300 \\ \mathrm{C} & -6.12747200 & -0.32921100 & -0.50696800 \\ \mathrm{C} & -5.00622500 & -0.68460100 & 0.23430500 \\ \mathrm{H} & -2.71694300 & 1.35780900 & -1.23142900 \\ \mathrm{H} & -4.71821100 & 1.99877100 & -2.54682800 \\ \mathrm{H} & -6.90029000 & 0.91319400 & -2.08353200 \\ \mathrm{H} & -7.08009300 & -0.80485200 & -0.30604800 \\ \mathrm{H} & -5.10144700 & -1.43961500 & 1.00391100 \\ \mathrm{C} & -2.61612100 & -1.29908400 & 1.97053600 \\ \mathrm{H} & -3.25971700 & -2.16241300 & 1.78312700 \\ \mathrm{H} & -1.60989300 & -1.67561100 & 2.15948800 \\ \mathrm{C} & -3.13461100 & -0.52019300 & 3.19833600 \\ \mathrm{H} & -3.12945300 & -1.17484100 & 4.07213600 \\ \mathrm{H} & -2.49687100 & 0.33801500 & 3.41817800 \\ \mathrm{H} & -4.15375200 & -0.15809700 & 3.05016400 \\ & & & \end{array}$

\section{(HMDS) ${ }_{2} \mathrm{Mg}\left\{\mathrm{O}=\mathrm{C}(\mathrm{Ph}) \mathrm{CH}_{2} \mathrm{CH}_{3}\right\}_{2}$ (III)}

\section{HF/6-31G ${ }^{*}$ optimization: $-2782.90096191 \mathrm{~h}$ B3LYP/6-311++G ${ }^{* *}:-2795.82726897 \mathrm{~h}$}

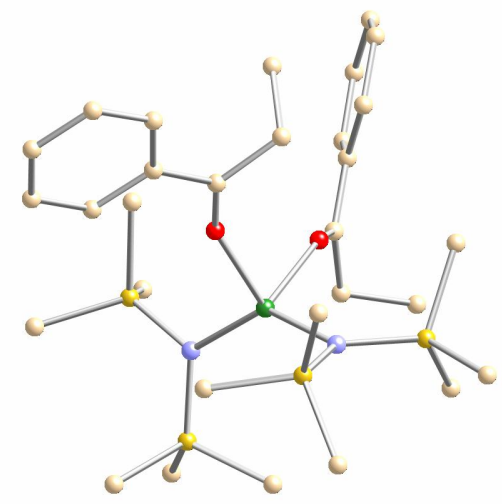

\section{Cartesian coordinates for the $\mathrm{HF} / 6-31 \mathrm{G}^{*}$ minimum:}

$\begin{array}{lrrr}\text { C } & -0.05949600 & 0.36353800 & 4.71257800 \\ \text { C } & -2.79973600 & 0.35115500 & 3.39938500 \\ \text { C } & -1.77621200 & -3.10333500 & 3.71118100 \\ \text { Si } & -0.92583000 & 0.32159000 & 3.01626700 \\ \text { C } & -0.63613300 & 2.06126400 & 2.29275400\end{array}$


$-0.53543400$

0.10869800

$-0.80858600$

$-1.94554200$

1.24300900

$-0.50167300$

$-0.55231600$

$-1.64445400$

$-0.01005600$

$-0.66041100$

0.03975700

0.43256400

$-1.50851300$

$-1.35367700$

$-2.50187500$

$-1.74417800$

$-2.90905500$

$-3.83688200$

$-2.44157800$

$-4.26423400$

5. 25182700

5.34710800

4. 60872900

4. 21655300

3. 68379300

2. 54577700

4.61570100

4.02365100

3. 16907200

2. 94062400

2. 24957300

1.29788400

1.90799000

4.67370300

3. 92718800

3. 24441300

3. 06359700

$-0.12932900$

$-0.39753000$

1. 91013600

0.38109700

$-1.62676400$

$-1.54091400$

$-2.60187000$

1.67950200

0.66966500

0.53641800 


$\begin{array}{lrrr}\mathrm{H} & -2.11622000 & 2.17696200 & -2.06249000 \\ \mathrm{H} & 2.94129600 & -2.52090600 & 1.07330600 \\ \mathrm{H} & 2.94052600 & -0.77927300 & 0.89059200 \\ \mathrm{H} & 4.35559200 & -1.70516100 & 0.45288600 \\ \mathrm{H} & -2.02552000 & -2.76377300 & -1.71416400 \\ \mathrm{H} & -1.06589900 & -4.15039000 & -2.16110300 \\ \mathrm{H} & -2.03494300 & -3.33206400 & -3.37390200 \\ \mathrm{H} & 2.54738000 & -4.48358500 & -1.31305400 \\ \mathrm{H} & 4.20696400 & -3.92289000 & -1.31228600 \\ \mathrm{H} & 3.29989800 & -3.95568100 & -2.80602600 \\ \mathrm{H} & -1.19112300 & 0.10284600 & -3.21323000 \\ \mathrm{H} & 0.24835700 & 0.06542600 & -4.21600800 \\ \mathrm{H} & -1.17686200 & -0.83225900 & -4.69491700 \\ \mathrm{H} & 3.69353400 & 0.20737900 & -2.12401000 \\ \mathrm{H} & 4.79806300 & -1.12246100 & -2.38847700 \\ \mathrm{H} & 3.45854900 & -0.86524200 & -3.48866500 \\ \mathrm{H} & 1.27040600 & -4.04334400 & -3.95826500 \\ \mathrm{H} & 1.93453100 & -2.55088700 & -4.59654900 \\ \mathrm{H} & 0.36942400 & -3.11481300 & -5.13442700 \\ \mathrm{C} & -3.73505400 & -1.01894900 & -0.03634400 \\ \mathrm{H} & -3.00944500 & -1.76931700 & 0.23928500 \\ \mathrm{H} & -4.28621300 & -0.76905000 & 0.86213400 \\ \mathrm{C} & -4.69828900 & -1.55799500 & -1.10660500 \\ \mathrm{H} & -4.18128300 & -1.77305100 & -2.03353700 \\ \mathrm{H} & -5.14203600 & -2.48141100 & -0.75355400 \\ \mathrm{H} & -5.49853300 & -0.86051100 & -1.32069200 \\ \mathrm{C} & 1.36740800 & 2.56063900 & -1.87008400 \\ \mathrm{H} & 2.19130300 & 2.66724900 & -2.56505500 \\ \mathrm{H} & 0.69145300 & 1.82480800 & -2.28098200 \\ \mathrm{C} & 0.64757000 & 3.90689400 & -1.68917400 \\ \mathrm{H} & 0.25459500 & 4.23918000 & -2.64358800 \\ \mathrm{H} & -0.18096500 & 3.81523600 & -0.99638400 \\ \mathrm{H} & 1.31237000 & 4.67609900 & -1.31506700 \\ & & & \\ & & & \\ & & & \end{array}$

\section{Cartesian coordinates for the B3LYP/6-311++G ${ }^{* *}$ minimum:}

$\begin{array}{lrrr}\text { C } & 0.30632200 & -0.30174500 & 4.69695900 \\ \text { C } & -2.55708600 & -0.16621100 & 3.70241400 \\ \text { C } & -1.62696300 & -3.61311700 & 3.36867300 \\ \text { Si } & -0.73960000 & -0.11481800 & 3.11787300 \\ \text { C } & -0.50955200 & 1.69640300 & 2.57750500 \\ \text { C } & 5.35884100 & 3.47962400 & 1.71702600 \\ \text { C } & 1.37558700 & -3.44552200 & 3.13848200 \\ \text { C } & 4.56833100 & 2.46998200 & 2.26827200 \\ \text { C } & -5.60529900 & 3.08228800 & -0.11701800 \\ \text { C } & 5.02430900 & 4.03375800 & 0.48199300 \\ \text { Si } & -0.23207400 & -2.95564900 & 2.24811000 \\ \text { C } & 3.44810800 & 2.01502600 & 1.58611400 \\ \text { C } & -4.94759100 & 1.87332800 & 0.08539900 \\ \text { N } & -0.34923000 & -1.25982800 & 1.87861600 \\ \text { C } & -5.04740600 & 4.04956400 & -0.95164900 \\ \text { C } & 3.90051200 & 3.58228800 & -0.20179800 \\ \text { C } & 3.09823000 & 2.56655700 & 0.34220000 \\ \text { C } & -3.71004500 & 1.62778500 & -0.52897300 \\ \text { C } & -3.82225300 & 3.81108400 & -1.57621400\end{array}$




\begin{tabular}{|c|c|c|c|}
\hline C & -0.34124100 & -4.00209000 & 0.67007700 \\
\hline $\mathrm{C}$ & -2.96398400 & 0.35847400 & -0.31314200 \\
\hline C & 1.89301200 & 2.05001000 & -0.35597100 \\
\hline C & -3.15349200 & 2.61337800 & -1.35852900 \\
\hline 0 & 1.29175800 & 1.08158200 & 0.11080100 \\
\hline 0 & -1.75076600 & 0.32968300 & -0.54123100 \\
\hline $\mathrm{Mg}$ & 0.08907000 & -0.64297100 & -0.02246900 \\
\hline $\mathrm{C}$ & 3.30439700 & -1.68655300 & 0.05135800 \\
\hline $\mathrm{N}$ & 0.89762800 & -1.46256000 & -1.72127700 \\
\hline Si & 2.59838800 & -1.78593900 & -1.70851500 \\
\hline $\mathrm{C}$ & -1.58921500 & -2.79024800 & -2.75778900 \\
\hline $\mathrm{C}$ & 3.09867900 & -3.52002100 & -2.30924200 \\
\hline $\mathrm{Si}$ & -0.09141500 & -1.67951400 & -3.12274000 \\
\hline C & -0.76297000 & -0.02497300 & -3.79298400 \\
\hline C & 3.60355800 & -0.54668900 & -2.75394800 \\
\hline $\mathrm{C}$ & 0.74638200 & -2.48387000 & -4.62915400 \\
\hline $\mathrm{H}$ & 0.09734100 & 0.51861600 & 5.39264700 \\
\hline $\mathrm{H}$ & 0.09653500 & -1.23705700 & 5.22444500 \\
\hline $\mathrm{H}$ & 1.37713900 & -0.28650100 & 4.47097000 \\
\hline $\mathrm{H}$ & -2.68108400 & 0.45884600 & 4.59419000 \\
\hline $\mathrm{H}$ & -2.89440700 & -1.17374000 & 3.95419700 \\
\hline $\mathrm{H}$ & -3.23323300 & 0.23156100 & 2.93864500 \\
\hline $\mathrm{H}$ & -1.60687500 & -3.18284600 & 4.37445000 \\
\hline $\mathrm{H}$ & -1.51973000 & -4.69802700 & 3.48024000 \\
\hline $\mathrm{H}$ & -2.61818600 & -3.42331900 & 2.94476100 \\
\hline $\mathrm{H}$ & -0.95045700 & 2.34280100 & 3.34452000 \\
\hline $\mathrm{H}$ & 0.54043100 & 1.97879700 & 2.47722700 \\
\hline $\mathrm{H}$ & -1.01079000 & 1.92651000 & 1.63297400 \\
\hline $\mathrm{H}$ & 6.23551300 & 3.83259500 & 2.24846000 \\
\hline $\mathrm{H}$ & 1.44013900 & -2.98770900 & 4.12969300 \\
\hline $\mathrm{H}$ & 1.42248800 & -4.53209300 & 3.27351800 \\
\hline $\mathrm{H}$ & 2.26546400 & -3.14176800 & 2.58142300 \\
\hline $\mathrm{H}$ & 4.82842400 & 2.03789200 & 3.22743300 \\
\hline $\mathrm{H}$ & -6.55258900 & 3.26919700 & 0.37504400 \\
\hline $\mathrm{H}$ & 5.64041600 & 4.81428000 & 0.05111700 \\
\hline $\mathrm{H}$ & 2.83009400 & 1.23079100 & 2.00317200 \\
\hline $\mathrm{H}$ & -5.39150300 & 1.13409000 & 0.73980700 \\
\hline $\mathrm{H}$ & -5.56649300 & 4.98713000 & -1.11591800 \\
\hline $\mathrm{H}$ & 3.65930800 & 4.01834700 & -1.16259300 \\
\hline $\mathrm{H}$ & -3.39233800 & 4.55961600 & -2.23165500 \\
\hline $\mathrm{H}$ & -0.11336500 & -5.04804400 & 0.90293700 \\
\hline $\mathrm{H}$ & -1.35005400 & -3.97919400 & 0.24490200 \\
\hline $\mathrm{H}$ & 0.34660000 & -3.67648400 & -0.11453100 \\
\hline $\mathrm{H}$ & -2.20388100 & 2.41663300 & -1.83838000 \\
\hline $\mathrm{H}$ & 2.94904700 & -2.51761600 & 0.66523200 \\
\hline $\mathrm{H}$ & 3.06524400 & -0.75832800 & 0.57515700 \\
\hline $\mathrm{H}$ & 4.39636900 & -1.76232600 & 0.00681800 \\
\hline $\mathrm{H}$ & -2.14926900 & -2.46093200 & -1.87942500 \\
\hline $\mathrm{H}$ & -1.26036400 & -3.81475700 & -2.55965000 \\
\hline $\mathrm{H}$ & -2.28175600 & -2.81970100 & -3.60645000 \\
\hline $\mathrm{H}$ & 2.47977100 & -4.29358500 & -1.84366300 \\
\hline $\mathrm{H}$ & 4.13996500 & -3.72035500 & -2.03237900 \\
\hline $\mathrm{H}$ & 3.02068800 & -3.63681800 & -3.39219500 \\
\hline $\mathrm{H}$ & -1.30014700 & 0.53868900 & -3.02637900 \\
\hline $\mathrm{H}$ & 0.05904300 & 0.60076900 & -4.15770300 \\
\hline $\mathrm{H}$ & -1.44940200 & -0.18591800 & -4.63178200 \\
\hline $\mathrm{H}$ & 3.51769300 & 0.47181200 & -2.3606440 \\
\hline
\end{tabular}




$\begin{array}{lrrr}\mathrm{H} & 4.66818000 & -0.80609100 & -2.75842500 \\ \mathrm{H} & 3.26598600 & -0.52487900 & -3.79476700 \\ \mathrm{H} & 1.00994000 & -3.52909200 & -4.44948300 \\ \mathrm{H} & 1.65067400 & -1.96052900 & -4.95281600 \\ \mathrm{H} & 0.04329800 & -2.46447100 & -5.46976500 \\ \mathrm{C} & -3.70043300 & -0.87604000 & 0.15020200 \\ \mathrm{H} & -2.95138700 & -1.63257400 & 0.38311300 \\ \mathrm{H} & -4.21396900 & -0.64638200 & 1.08852600 \\ \mathrm{C} & -4.72296600 & -1.39630000 & -0.88021600 \\ \mathrm{H} & -4.24486700 & -1.62553900 & -1.83372400 \\ \mathrm{H} & -5.17689800 & -2.31596500 & -0.50523600 \\ \mathrm{H} & -5.52032200 & -0.67402700 & -1.06373600 \\ \mathrm{C} & 1.37512600 & 2.72674400 & -1.60490500 \\ \mathrm{H} & 2.20425800 & 2.96046500 & -2.27717000 \\ \mathrm{H} & 0.73412400 & 2.00670300 & -2.11390000 \\ \mathrm{C} & 0.58683000 & 4.01095200 & -1.27629300 \\ \mathrm{H} & 0.19367400 & 4.44721900 & -2.19744900 \\ \mathrm{H} & -0.25466100 & 3.79524900 & -0.61470800 \\ \mathrm{H} & 1.21404800 & 4.75998900 & -0.78867800\end{array}$

\section{$\mathrm{O}=\mathrm{C}(\mathrm{Ph}) \mathrm{CH}_{2} \mathrm{CH}_{3}(\mathbf{I V})$}

HF/6-31G* optimization: -421.509133551 h B3LYP/6-311++G**: $-424.324161360 \mathrm{~h}$

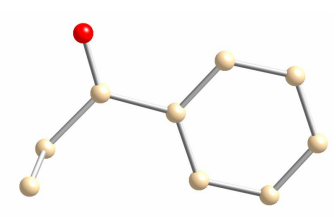

\section{Cartesian coordinates for the $\mathrm{HF} / 6-31 \mathrm{G}^{*}$ minimum:}

$\begin{array}{lrrr}\text { C } & 1.93232500 & -1.42323900 & -0.18230500 \\ \mathrm{C} & 0.59300100 & -1.08901900 & -0.30809600 \\ \mathrm{C} & 2.86398000 & -0.44437800 & 0.11595700 \\ \mathrm{C} & 0.17388300 & 0.22577800 & -0.13620200 \\ \mathrm{C} & 2.45675900 & 0.87111800 & 0.28951700 \\ \mathrm{C} & -1.26136300 & 0.65327100 & -0.25969400 \\ \mathrm{C} & 1.12164100 & 1.20241500 & 0.16462600 \\ \mathrm{O} & -1.55820700 & 1.80477200 & -0.12531200 \\ \mathrm{H} & 2.24404900 & -2.44317400 & -0.31775600 \\ \mathrm{H} & -0.11294600 & -1.86375900 & -0.54019200 \\ \mathrm{H} & 3.90331400 & -0.70326000 & 0.21286000 \\ \mathrm{H} & 3.17942300 & 1.63268500 & 0.52109100 \\ \mathrm{H} & 0.79328200 & 2.21524900 & 0.29577400 \\ \mathrm{C} & -2.34795300 & -0.37653100 & -0.51754300 \\ \mathrm{H} & -3.18021700 & 0.16285200 & -0.95112500 \\ \mathrm{H} & -2.02305300 & -1.11773100 & -1.23871800 \\ \mathrm{C} & -2.79700100 & -1.05862900 & 0.78284500 \\ \mathrm{H} & -3.15610200 & -0.32172100 & 1.49258300 \\ \mathrm{H} & -3.60477400 & -1.75392500 & 0.58223300 \\ \mathrm{H} & -1.98895600 & -1.61010700 & 1.25111600\end{array}$


Cartesian coordinates for the B3LYP/6-311++G ${ }^{* *}$ minimum:

$\begin{array}{lrrr}\mathrm{C} & 1.93988800 & -1.42918200 & -0.18708600 \\ \mathrm{C} & 0.59350600 & -1.09441300 & -0.31572500 \\ \mathrm{C} & 2.87740100 & -0.44495200 & 0.11879300 \\ \mathrm{C} & 0.16951400 & 0.22948400 & -0.13871200 \\ \mathrm{C} & 2.46580500 & 0.87742000 & 0.29668000 \\ \mathrm{C} & -1.26711700 & 0.65297300 & -0.26501100 \\ \mathrm{C} & 1.12340200 & 1.21089200 & 0.16917000 \\ \mathrm{O} & -1.57543700 & 1.82431200 & -0.13657900 \\ \mathrm{H} & 2.25615700 & -2.45668600 & -0.32658100 \\ \mathrm{H} & -0.12002900 & -1.87283300 & -0.55534600 \\ \mathrm{H} & 3.92519300 & -0.70607900 & 0.21767200 \\ \mathrm{H} & 3.19384300 & 1.64487400 & 0.53423300 \\ \mathrm{H} & 0.78450600 & 2.23085700 & 0.30278300 \\ \mathrm{C} & -2.34617100 & -0.39189500 & -0.51501600 \\ \mathrm{H} & -3.18677500 & 0.13665100 & -0.96822300 \\ \mathrm{H} & -2.00374900 & -1.14742800 & -1.22688600 \\ \mathrm{C} & -2.80199800 & -1.06444300 & 0.79411200 \\ \mathrm{H} & -3.17368000 & -0.31728500 & 1.49915000 \\ \mathrm{H} & -3.60991700 & -1.77262600 & 0.59504200 \\ \mathrm{H} & -1.98743500 & -1.60924500 & 1.27756200\end{array}$

\section{$(\mathrm{HMDS}){ }_{2} \mathrm{Mg}\left\{\mathrm{O}=\mathrm{C}\left({ }^{\mathrm{t}} \mathrm{Bu}\right) \mathrm{Ph}\right\}(\mathbf{V})$}

HF/6-31G * optimization: $-2439.44119229 \mathrm{~h}$ B3LYP/6-311++G ** : $-2450.13466238 \mathrm{~h}$

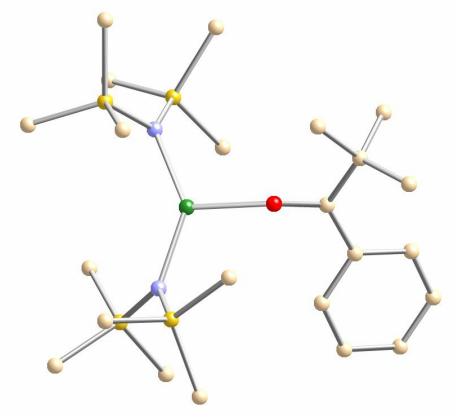

\section{Cartesian coordinates for the $\mathrm{HF} / 6-31 \mathrm{G}^{*}$ minimum:}

$\begin{array}{lrrr}\text { C } & 1.08823600 & 3.76500900 & 2.54780700 \\ \text { C } & -3.53620500 & -2.52186300 & 2.37142300 \\ \text { C } & -0.91230600 & 1.50562300 & 2.79903000 \\ \text { C } & 2.87492400 & -0.31912200 & 2.52600700 \\ \text { C } & -1.04620400 & -2.64786200 & 2.39292500 \\ \text { C } & 4.40472700 & -2.57543700 & 1.18555100 \\ \text { Si } & -0.26096000 & 2.82993300 & 1.59159200 \\ \text { C } & -2.28407500 & -2.63604700 & 1.48046400 \\ \text { C } & -1.70897000 & 4.04282600 & 1.36109000 \\ \text { Si } & 3.47102800 & -0.95830200 & 0.83449200 \\ \text { C } & -2.28635800 & -3.95442300 & 0.67337000\end{array}$




\begin{tabular}{|c|c|c|}
\hline 600 & 0.29282700 & 200 \\
\hline 2.17551000 & -1.44774100 & 0.50908300 \\
\hline 400 & -0.89162200 & 978900 \\
\hline .28136700 & 2.11086900 & 2 \\
\hline 0.61773300 & 0.14037800 & 0.06647 \\
\hline 1.47025300 & 4.70193500 & 0.95617200 \\
\hline .31404500 & -0.91336300 & -0.298350 \\
\hline .12434600 & -1.12908700 & -0.2 \\
\hline ( & -1.65253100 & \\
\hline 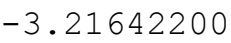 & 0.41017500 & \\
\hline .633278 & 3.02933300 & -1.28863 \\
\hline .43978000 & -1.08705500 & -1.42546 \\
\hline 260100 & 0.98056400 & -1.489997 \\
\hline 35100 & -4 & -1 \\
\hline 0 & 0.2 & -1 \\
\hline 400 & 2.0 & $-2+2-1$ \\
\hline .04986300 & -2.19711900 & -1.55703 \\
\hline 203800 & 3.39472400 & -2.32512 \\
\hline & -1. & \\
\hline 0.4 & -1.5 & -2 . \\
\hline 0.70 & 4.1 & 3 \\
\hline 1.94867800 & 3.1 & 2.75300 \\
\hline 1.4508 & 4.63415700 & 2.00768 \\
\hline 934400 & -3.32115100 & 3.1038950 \\
\hline & -1 & \\
\hline$\cdot 4$ & -2.5 & 1.8 \\
\hline$-1 \cdot 1$ & 1.974 & 3.74 \\
\hline .18542900 & 0.72708900 & 3.0231800 \\
\hline-1.81615400 & 1.02516000 & 2.432959 \\
\hline 700 & -0.2 & 3. \\
\hline & -1 . & \\
\hline 2.3 & 0. & 2 . \\
\hline-1.12095100 & -3.487 & $3.0^{\circ}-x$ \\
\hline-0.98134400 & -1.74320200 & 2.98535 \\
\hline .12929900 & -2.75184 & $1.82901 \mathrm{~s}$ \\
\hline 400 & -2 . & 574 \\
\hline 3.7 & -3. & 1 . \\
\hline 4.8 & -2.9 & 0 . \\
\hline 783900 & $4.42-2 x-1$ & 2.3237020 \\
\hline-1.44630100 & 4.900043 & 0.7466210 \\
\hline 566723 & 3.5 & 0.8 \\
\hline & -4 . & 952 \\
\hline 0 & -4 & 0 . \\
\hline .40574100 & -4.0 & 0 . \\
\hline 59831200 & 0.3 & 38990 \\
\hline 33028500 & 1.28758000 & 0.1134300 \\
\hline 7800 & 96300 & -0.7559000 \\
\hline 1093300 & 4.5 & -0.4252340 \\
\hline 200 & & -0.376143 \\
\hline 60175400 & 49200 & -1.8994260 \\
\hline .55374500 & -2.67260900 & -0.3506060 \\
\hline & 0.99654600 & -0.4718880 \\
\hline 581800 & -1.67476800 & -1.7018470 \\
\hline 3544 & 2.00294100 & -1.8072050 \\
\hline & -4.23658800 & \\
\hline & -4.39323000 & \\
\hline 0100 & -4.65454000 & -1.9558710 \\
\hline
\end{tabular}




$\begin{array}{lrrr}\mathrm{H} & -6.11521600 & 0.67049900 & -2.43456800 \\ \mathrm{H} & 2.74940100 & 1.83636800 & -1.96831600 \\ \mathrm{H} & 1.38477300 & 1.16824600 & -2.84004900 \\ \mathrm{H} & 2.04095400 & 2.71173800 & -3.31011400 \\ \mathrm{H} & -1.66463400 & 3.95874800 & -1.76588300 \\ \mathrm{H} & -1.39660300 & 2.47582500 & -2.66544400 \\ \mathrm{H} & -0.67505000 & 3.97448600 & -3.21239500 \\ \mathrm{H} & 4.45297000 & -2.21372400 & -2.31129000 \\ \mathrm{H} & 3.54562600 & -0.98177300 & -3.16304100 \\ \mathrm{H} & 3.38833900 & -2.66855300 & -3.62401300 \\ \mathrm{H} & -0.44233700 & -2.18881700 & -2.01690500 \\ \mathrm{H} & 0.32427500 & -0.87378800 & -2.89176500 \\ \mathrm{H} & 0.47537800 & -2.51070800 & -3.46540600\end{array}$

\section{Cartesian coordinates for the B3LYP/6-311++G ${ }^{* *}$ minimum:}

$\begin{array}{lrrr}\text { C } & 0.98793600 & 3.79455600 & 2.55078900 \\ \mathrm{C} & -3.53123400 & -2.51399000 & 2.39350900 \\ \mathrm{C} & -0.90174200 & 1.43961900 & 2.81038600 \\ \mathrm{C} & 2.97092300 & -0.04336900 & 2.41503900 \\ \mathrm{C} & -1.03298600 & -2.67659200 & 2.42468800 \\ \mathrm{C} & 4.41252600 & -2.47458500 & 1.28536400 \\ \mathrm{Si} & -0.33672900 & 2.80859000 & 1.61495300 \\ \mathrm{C} & -2.27170100 & -2.65552100 & 1.50788400 \\ \mathrm{C} & -1.82702300 & 3.96878900 & 1.40367900 \\ \mathrm{Si} & 3.54074300 & -0.86089700 & 0.79598500 \\ \mathrm{C} & -2.29607300 & -3.98754000 & 0.71044900 \\ \mathrm{C} & 4.85502100 & 0.27830700 & 0.03896200 \\ \mathrm{C} & -2.14444700 & -1.48169500 & 0.51703700 \\ \mathrm{O} & -1.04884100 & -0.92072900 & 0.41526400 \\ \mathrm{~N} & 0.21949800 & 2.11358100 & 0.12564600 \\ \mathrm{Mg} & 0.64697500 & 0.16297300 & 0.05830200 \\ \mathrm{C} & 1.43505000 & 4.70234800 & -0.94110500 \\ \mathrm{C} & -3.26997100 & -0.96363500 & -0.31179800 \\ \mathrm{~N} & 2.16468900 & -1.08646800 & -0.23286600 \\ \mathrm{C} & -4.41991600 & -1.70223600 & -0.63956700 \\ \mathrm{C} & -3.15243100 & 0.35010600 & -0.80347800 \\ \mathrm{Si} & 0.55378400 & 3.05951700 & -1.29344700 \\ \mathrm{C} & -5.41166900 & -1.14833000 & -1.44108200 \\ \mathrm{C} & -4.15559200 & 0.90702300 & -1.58601100 \\ \mathrm{C} & 1.93324600 & -4.02866400 & -0.93973400 \\ \mathrm{C} & -5.28590100 & 0.15803000 & -1.91110500 \\ \mathrm{C} & 1.66222100 & 2.09804500 & -2.49558700 \\ \mathrm{Si} & 2.07395800 & -2.22663700 & -1.52863700 \\ \mathrm{C} & -1.02075900 & 3.48614800 & -2.27320400 \\ \mathrm{C} & 3.55217400 & -2.14679000 & -2.71537100 \\ \mathrm{C} & 0.51824400 & -1.90503100 & -2.58071500 \\ \mathrm{H} & 0.59394900 & 4.15408200 & 3.50810000 \\ \mathrm{H} & 1.86943200 & 3.18293200 & 2.76634000 \\ \mathrm{H} & 1.32549000 & 4.66703100 & 1.98545700 \\ \mathrm{H} & -3.52996400 & -3.31450600 & 3.13773900 \\ \mathrm{H} & -3.52676800 & -1.56253000 & 2.93199700 \\ \mathrm{H} & -4.46421300 & -2.57877200 & 1.83550400 \\ \mathrm{H} & -1.17998400 & 1.88743800 & 3.77024100 \\ \mathrm{H} & -0.11402100 & 0.70895600 & 3.02468300 \\ & & & \\ & & & \\ & & & \end{array}$




$\begin{array}{lrrr}\mathrm{H} & -1.77707200 & 0.90121500 & 2.43661000 \\ \mathrm{H} & 3.82496100 & 0.14242300 & 3.07488600 \\ \mathrm{H} & 2.26983200 & -0.67983000 & 2.96589800 \\ \mathrm{H} & 2.49313100 & 0.92845500 & 2.24785700 \\ \mathrm{H} & -1.11595100 & -3.52003500 & 3.11451700 \\ \mathrm{H} & -0.96058500 & -1.76194100 & 3.01667100 \\ \mathrm{H} & -0.10913700 & -2.78765700 & 1.85642300 \\ \mathrm{H} & 5.22977200 & -2.26310200 & 1.98348200 \\ \mathrm{H} & 3.72514800 & -3.16944300 & 1.77727700 \\ \mathrm{H} & 4.84805600 & -2.99122600 & 0.42463900 \\ \mathrm{H} & -2.17017900 & 4.33092700 & 2.37899300 \\ \mathrm{H} & -1.58149000 & 4.84886200 & 0.80124000 \\ \mathrm{H} & -2.67050900 & 3.46381000 & 0.92240500 \\ \mathrm{H} & -2.29873300 & -4.81776600 & 1.42155700 \\ \mathrm{H} & -3.17096300 & -4.09730500 & 0.07008300 \\ \mathrm{H} & -1.40501100 & -4.08693600 & 0.08641400 \\ \mathrm{H} & 5.70763500 & 0.41261000 & 0.71374400 \\ \mathrm{H} & 4.44040200 & 1.26925400 & -0.17180200 \\ \mathrm{H} & 5.23743100 & -0.12418000 & -0.90398500 \\ \mathrm{H} & 2.37932700 & 4.54520900 & -0.41150100 \\ \mathrm{H} & 0.82588100 & 5.38396000 & -0.33992600 \\ \mathrm{H} & 1.66273200 & 5.21518900 & -1.88188700 \\ \mathrm{H} & -4.54279900 & -2.71826500 & -0.29711300 \\ \mathrm{H} & -2.27521800 & 0.93640200 & -0.55572400 \\ \mathrm{H} & -6.28372000 & -1.73797800 & -1.69861300 \\ \mathrm{H} & -4.05149300 & 1.92417500 & -1.94461200 \\ \mathrm{H} & 1.10526800 & -4.15079800 & -0.23384800 \\ \mathrm{H} & 2.84304100 & -4.36131800 & -0.43270400 \\ \mathrm{H} & 1.75780600 & -4.70739500 & -1.78189900 \\ \mathrm{H} & -6.06557400 & 0.58980600 & -2.52842500 \\ \mathrm{H} & 2.61650100 & 1.80505100 & -2.04945100 \\ \mathrm{H} & 1.18380200 & 1.19223200 & -2.88188600 \\ \mathrm{H} & 1.88409700 & 2.72677000 & -3.36469500 \\ \mathrm{H} & -1.73696600 & 4.04974700 & -1.66797300 \\ \mathrm{H} & -1.52192300 & 2.57971700 & -2.62922400 \\ \mathrm{H} & -0.78095800 & 4.09322600 & -3.15313000 \\ \mathrm{H} & 4.49429700 & -2.37824800 & -2.20846700 \\ \mathrm{H} & 3.65200700 & -1.14985800 & -3.15541800 \\ \mathrm{H} & 3.43899100 & -2.86506100 & -3.53484400 \\ \mathrm{H} & -0.41124800 & -2.04240900 & -2.01926500 \\ \mathrm{H} & 0.50444500 & -0.90094700 & -3.01698800 \\ \mathrm{H} & & & \\ & & & \end{array}$

